\title{
Flowers and mycorrhizal roots - closer than we think?
}

\author{
Eva Nouri and Didier Reinhardt \\ Department of Biology, University of Fribourg, Fribourg, Switzerland
}

\begin{abstract}
Roots and flowers are formed at the extreme ends of plants and they differ in almost every aspect of their development and function; even so, they exhibit surprising molecular commonalities. For example, the calcium and calmodulin-dependent protein kinase (CCaMK) plays a central role in root symbioses with fungi and bacteria, but is also highly expressed in developing anthers. Moreover, independent evidence from transcriptomics, phylogenomics, and genetics reveals common developmental elements in root symbioses and reproductive development. We discuss the significance of these overlaps, and we argue that an integrated comparative view of the two phenomena will stimulate research and provide new insight, not only into shared components, but also into the specific aspects of anther development and root symbioses.
\end{abstract}

Roots and flowers: closer than apples and oranges? Most plants engage in arbuscular mycorrhiza (AM), mutualistic associations with fungi from the order Glomeromycota [1-3]. The wide distribution of this interaction among land plants, and its predominance in almost all ecological niches, suggests that this symbiosis provides diverse important services to the host [4]. Establishment of AM requires a signaling cascade in the host, referred to as the common symbiosis signaling pathway (CSSP) because it is shared with the nitrogen-fixing nodule symbiosis between legumes and rhizobia [2,5].

One of the central elements of the CSSP is CCaMK, which is required for infection by AM fungi and rhizobia [6]. Constitutively active forms of CCaMK are sufficient to trigger the formation of nodules in the absence of rhizobia or of any exogenous stimulus, hence demonstrating the central role of CCaMK in nodulation [7,8]. Interestingly, CCaMK was originally identified and characterized not in roots, but in the anthers of flowers, hence the provocative question: does pollen development involve mechanisms shared with root symbiosis? What might the two have in common? Based on several lines of evidence, it may be related to signaling and cell wall biosynthesis.

Pollen grains represent highly-resistant capsules which protect the sensitive sperm cells (or their progenitor cell) against harsh conditions such as desiccation and

Corresponding author: Reinhardt, D. (didier.reinhardt@unifr.ch).

Keywords: symbiosis; arbuscular mycorrhiza; pollen; glycerol-3-phosphate acyltransferase; GPAT.
UV irradiation. The wall of the capsule consists of a sturdy multi-layered cell wall [9] that is coated with one of the most resistant biological materials, sporopollenin, that consists of a hydrophobic polymer of fatty acids and their derivatives [10]. Owing to its extreme resistance and wide distribution in nature, pollen is one of the best fossil indicators for evolutionary and climatic studies over large spatial and temporal scales [11].

At the other end of the spectrum regarding longevity reside the ephemeral arbuscules, the feeding structures of AM fungi that have a lifetime of only a few days [12,13]. Nevertheless, surprisingly, the development of arbuscular mycorrhiza requires a similar lipid-related pathway in the plant host as that involved in pollen wall synthesis. However, in the case of symbiosis, there is no evidence for the formation of an extracellular polymer by the plant. Instead, the non-polymerized lipidic intermediates of the pathway are thought to function as signals between the plant host and the fungal endosymbiont [14-16]. Moreover, genomic and genetic analyses reveal further unexpected parallels between root symbiosis and pollen development.

We discuss here recent highlights in these so far unrelated research domains, explore the significance of overlaps between the involved pathways, and suggest that a scientific crosstalk between the two research fields could potentially contribute to advancing both fields in the coming years.

\section{The precedent: the case of CCaMK}

CCaMK was first isolated from lilly (Lilium longiflorum) anthers [17], and subsequently biochemically characterized in lilly, tobacco (Nicotiana tabacum), maize (Zea mays), and pea (Pisum sativum) [18-25]. The combination of a calmodulin-binding domain and three EF hands in CCaMK is unique (Box 1) and sets it apart from the calcium-dependent protein kinases (CDPKs) [6]. In lilly and tobacco anthers, CCaMK is expressed at highest levels during the meiotic phase of pollen development, in the meiocytes as well as in the tapetum, the cell layer that feeds the developing pollen [18,26].

The function of CCaMK has long remained elusive, and when the first knockout mutants of ccamk were identified in Medicago truncatula their phenotype was not related to flowers but, surprisingly, to the roots, which were defective in the development of both AM and nitrogen-fixing nodules $[27,28]$. Subsequent work showed that the AM-related function of CCaMK and its expression in flowers are conserved in rice (Oryza sativa) [29,30]. The function of CCaMK in symbiosis is thought to be the decoding of a 
CCaMK is a protein kinase that can bind three calcium ions with the EF hands (red blocks) in its C-terminal domain (Figure I). This domain, which is evolutionarily related to calmodulin (although one EF hand is nonfunctional, depicted in pink), is shared with the calcium-binding domain of CDPKs [70]. However, in contrast to CDPKs, CCaMK has in addition a calmodulin-binding domain (CBD) which is essential for proper regulation $[6,71]$. CCaMK, which is encoded by a single-copy gene in most plants, is highly conserved in species that can undergo
AM and/or root nodule symbiosis [34,72]; however, Arabidopsis thaliana and other non-symbiotic species have lost it. For phylogenetic analysis, MtCCaMK was used as a bait to identify the three closest homologs from Medicago truncatula (Mt), A. thaliana (At), Solanum lycopersicum (SI), Cucumis sativus (Cs), Oryza sativa (Os), Zea mays $(\mathrm{Zm})$, and Sorghum bicolor $(\mathrm{Sb})$ by PBLAST at NCBI. Phylograms were produced as described using the software package at www.phylogeny.fr [73]. Support for branch separation is indicated with bootstrap values for 100 replicates.

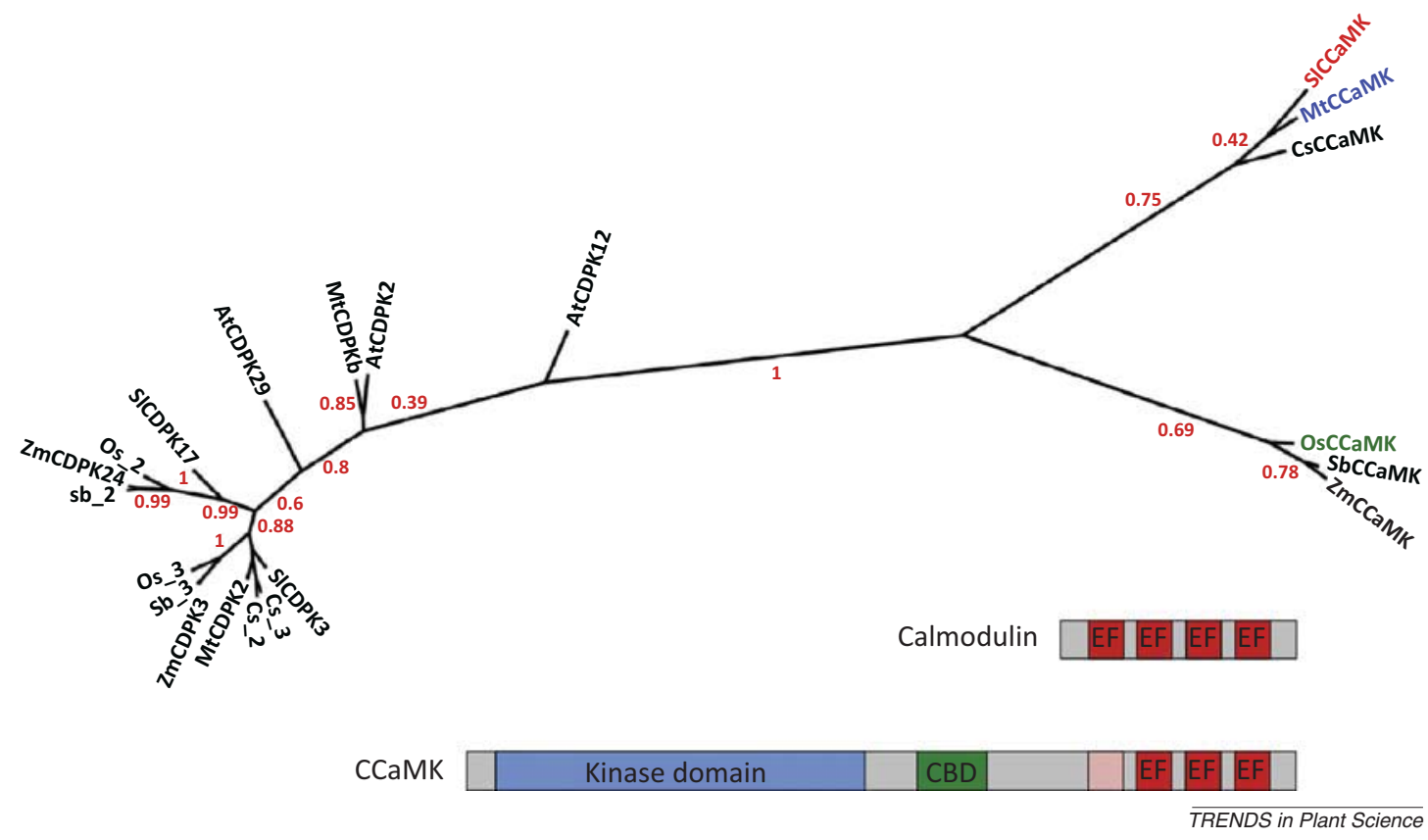

Figure I. Phylogram of plant CCaMK proteins.

calcium signal (calcium spiking) in root cells that have perceived AM fungal or rhizobial signals (reviewed in [6]). Interestingly, mutants defective in two other components of the CSSP that act upstream of calcium spiking, nucleoporin 85 (NUP85) and NUP133, also point to an overlap between root symbiosis and reproduction because they exhibit fertility defects [31-33].

\section{A lipid-related pathway shared between AM and flower development}

To explore potentially shared regulatory pathways in flower development and root symbiosis in a systematic fashion, a comparison of the respective transcriptomes can be used as an indicator for common developmental and regulatory pathways. Because the well-characterized model species Arabidopsis thaliana does not engage in root symbioses, we chose the standard symbiosis model species $M$. truncatula which offers excellent bioinformatics tools with the M. truncatula Gene Expression Atlas (MtGEA; http:// mtgea.noble.org/v3).

By comparing gene expression in flowers with vegetative buds, and applying a threefold induction cutoff, a total of 151 flower-induced genes were identified. In a second step, these flower-related genes were assessed in relation to three recently established AM-related criteria [34]: (i) threefold induction in mycorrhizal roots, (ii) a significant
AM-related pattern of sequence conservation in the coding region, and (iii) predicted AM-related regulatory sequences in their promoters. Surprisingly, 81 of the 151 flowerinduced genes $(53.6 \%)$ were found in at least one of these three AM-related categories. Considering the 20 genes with the highest induction levels in AM (Table 1), 10 (50\%) exhibited a significant $(P<0.05)$ AM-related conservation pattern as defined in [34] or were entirely missing from $A$. thaliana (indicated with asterisks in Table 1). This is considerably more than the $28.5 \%$ of genes $(n=1334)$ with an AM-related conservation pattern when 4684 ubiquitous housekeeping genes were considered (see Table S5 in [34]). This suggests that these 10 flower-induced genes have been under selection for AM-related functions, and therefore may have overlapping functions in flowers and in AM symbiosis.

The gene with the highest induction ratio in AM is a glycerol-3-phosphate acyltransferase (GPAT) (Table 1), known as REQUIRED FOR ARBUSCULAR MYCORRHIZA2 (RAM2), based on its AM-defective mutant phenotype [16]. GPAT catalyzes the transfer of fatty acids onto glycerol-3-phosphate [35,36]. This is the first committed step in the biosynthesis of phospholipids (membrane lipids) and triacylglycerols (storage lipids), and of extracellular lipid polyesters such as cutin and suberin (Box 2). An important distinction in these pathways is that the precursors of 
Table 1. Genes commonly induced in AM symbiosis and in flowers of Medicago truncatula ${ }^{\mathrm{a}}$

\begin{tabular}{|c|c|c|c|c|c|c|c|}
\hline & GenelD & Probeset & LCM & AM & Flo/Bud & Annotation & At homolog \\
\hline $1^{\mathrm{b}}$ & MTR_1g040500.1 & Mtr.36944.1.S1_at & 321.9 & 45.1 & 75.2 & ER glycerol-phosphate acyltransferase (RAM2) & At2g38110 \\
\hline $2^{\mathrm{b}}$ & MTR_6g073040.1 & Mtr.41728.1.S1_at & 199.3 & 241.4 & 22.8 & Putative uncharacterized protein (CASP1-like) & At2g39530 \\
\hline $3^{b}$ & MTR_8g091690.1 & Mtr.12170.1.S1_at & 58.1 & 93.7 & 30.7 & Cytochrome P450 (CYP71A1; ferulate 5-hydroxylase) & At4g36220 \\
\hline 4 & MTR_8g087810.1 & Mtr.46057.1.S1_at & 47.7 & 31.3 & 3.2 & Nitrate/chlorate transporter & At1g59740 \\
\hline 5 & MTR_8g038210.1 & Mtr.14183.1.S1_at & 27.5 & 2.5 & 11.1 & Annexin & At5g12380 \\
\hline 6 & MTR_8g074530.1 & Mtr.43640.1.S1_at & 20.5 & 1.6 & 3.9 & Putative uncharacterized protein & At4g35690 \\
\hline 7 & MTR_4g122750.1 & Mtr.38447.1.S1_at & 14.0 & 1.1 & 19.5 & Pectinesterase inhibitor & At1g47960 \\
\hline 8 & MTR_3g020970.1 & Mtr.30156.1.S1_at & 11.8 & 6.0 & 7.5 & Subtilisin inhibitor & At2g38900 \\
\hline 9 & MTR_5g017550.1 & Mtr.44890.1.S1_at & 11.1 & 0.9 & 26.2 & Calcium-binding protein CML24 & At2g15680 \\
\hline $10^{\mathrm{b}}$ & MTR_5g018610.1 & Mtr.29593.1.S1_at & 8.4 & 29.6 & 4.0 & Putative uncharacterized protein & no homolog \\
\hline $11^{\mathrm{b}}$ & MTR_2g100350.1 & Mtr.8968.1.S1_at & 7.8 & 0.4 & 27.9 & Pyridoxal phosphate phosphatase $\mathrm{PHOSPHO} 2$ & At1g17710 \\
\hline $12^{\mathrm{b}}$ & MTR_7g083570.1 & Mtr.22351.1.S1_at & 7.0 & 0.5 & 3.5 & GEM-like protein & At5g13200 \\
\hline 13 & MTR_7g082570.1 & Mtr.12958.1.S1_at & 6.7 & 1.3 & 5.2 & Class I glutamine amidotransferase & At5g38200 \\
\hline $14^{\mathrm{b}}$ & MTR_6g009260.1 & Mtr.17352.1.S1_at & 6.4 & 10.7 & 3.9 & Synaptojanin-1 & At3g63240 \\
\hline 15 & MTR_3g084200.1 & Mtr.20624.1.S1_at & 6.1 & 0.8 & 3.5 & Auxin-induced protein-like protein & At4g38840 \\
\hline 16 & MTR_2g101560.1 & Mtr.12373.1.S1_at & 5.1 & 0.9 & 14.4 & Nitrate/peptide transporter & At1g52190 \\
\hline 17 & MTR_8g094740.1 & Mtr.44450.1.S1_at & 4.7 & 1.8 & 4.6 & Glutamyl-tRNA(GIn) amidotransferase subunit $A$ & At5g64440 \\
\hline $18^{\mathrm{b}}$ & MTR_3g061170.1 & Mtr.43887.1.S1_at & 4.5 & 0.8 & 3.8 & Chaperone protein dnaJ & At3g13310 \\
\hline $19^{\mathrm{b}}$ & MTR_7g012330.1 & Mtr.43426.1.S1_at & 4.1 & 0.6 & 52.6 & Cytochrome P450 (CYP71B37) & At4g31500 \\
\hline $20^{\mathrm{b}}$ & MTR_3g088460.1 & Mtr.17288.1.S1_at & 4.0 & 0.7 & 7.4 & Metal transporter & At1g15960 \\
\hline
\end{tabular}

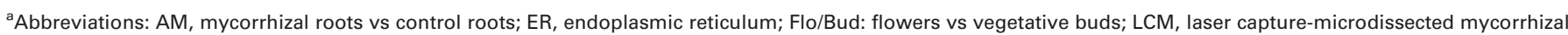
cortex cells vs non-colonized cells.

${ }^{\mathrm{b}}$ Genes that exhibit a significant $(P<0.05)$ AM-related conservation pattern as defined in [34], or that were lost in Arabidopsis.

membrane and storage lipids are first acylated at the sn1-position, whereas the precursors of the extracellular polyesters are acylated at the sn2-position [35,36] (Box 2).

Plants have typically 8-10 GPATs distributed over six subfamilies (Figure 1). Based on its relative proximity to GPAT4 to GPAT8 (Figure 1), RAM2 is predicted to have sn2-acylation activity. Furthermore, RAM2 shares conserved amino acid residues with GPAT4, GPAT6, and GPAT8 that are required for phosphatase activity [16,37]. This activity releases the phosphate group from the $s n 3$-position, thereby generating precursors for cutin biosynthesis (Box 2). Based on this collective evidence, RAM2 in pollen may be involved in the synthesis of an extracellular lipidic polymer, perhaps sporopollenin.

What is the role of RAM2 in AM symbiosis? Lipidic polyesters function primarily as barriers for water and soluble molecules [38], a function that is not immediately obvious in mycorrhizal roots where facilitated nutrient transfer is the central symbiotic function. Based on the fact that ram 2 mutants show extremely low levels of AM fungal contact points, it has been proposed that the products of the GPAT activity of RAM2 may function as signals from the host to stimulate fungal infection [14-16].

The second gene in the list of shared AM- and flowerinduced genes (Table 1) encodes a predicted CASP1-like protein. CASPs, which are encoded by a gene family in Arabidopsis, are membrane proteins that localize to the Casparian strip, and are required for its formation [39]. The third gene in the list of AM- and flower-induced genes is a cytochrome P450 belonging to the subfamily CYP71, and a ferulate 5-hydroxylase (FAH) is the closest homolog in Arabidopsis (Table 1). FAH is involved in the biosynthesis of lignin by oxidizing one of its aromatic constituents [40,41]. Because both functions, CASP1-like and CYP71, appear to be related to cell wall modifications, it is possible that the apoplastic symbiotic interface of mycorrhizal roots and the pollen wall share some unknown features. However, it should be borne in mind that, despite their tentative annotation based on Arabidopsis, the functions of CASP1-like and of CYP71 in root symbiosis and pollen development are elusive and will require functional analysis by reverse genetics approaches.

\section{Recognition in $A M$ and in pollination may involve a common mechanism}

At a superficial level, the interaction between fungal hyphae and roots, and between pollen tubes and the stigma, exhibit similar aspects. In both cases, a tubular structure (fungal hyphae and pollen tubes, respectively) penetrate plant tissues (the root surface and the stigma, respectively). A parallel at the molecular level involves the specific recognition mechanisms involved in the two interactions which, however, lead to opposite downstream consequences. In $\mathrm{AM}$, the recognition of symbiosis-specific signals and the activation of the CSSP are prerequisites for a compatible interaction $[2,3,42]$. By contrast, plants use the recognition of pollen-specific signals to identify their own pollen and to reject it, a phenomenon known as self-incompatibility (SI) [43].

There are two different mechanisms of SI, gametophytic and sporophytic SI, depending on whether recognition involves factors encoded by the haploid pollen grain (the gametophyte) or factors encoded by the diploid anther tissue of the sporophyte (in particular the tapetum) of the plant that produced the pollen. In the well-characterized sporophytic SI of the Brassicaceae (including the genus Arabidopsis), the diploid anther tissues produce a protein, the S-locus cysteine-rich (SCR) protein, that is deposited on the pollen coat during pollen development [44]. This extracellular protein is recognized in the stigma 
GPATs mediate the first dedicated step in the biosynthesis of membrane lipids, storage lipids, and extracellular lipid polyesters (cutin and suberin) (Figure I). In general, GPATs use acyl-CoA as a substrate, from which they transfer the acyl chain onto glycerol3-phosphate. In the case of membrane and storage lipids, the acyl group is transferred to the sn 1-position, resulting in lysophosphatidic acid (1-acyl-LPA), followed by the transfer of a second acyl chain to the sn2-position. Cutin and suberin precursors are formed by the transfer of the acyl chain to the sn2-position (2-acyl-LPA). In the case of cutin biosynthesis, the product of the acyltransferase reaction is simultaneously dephosphorylated, resulting in monoacylglycerol (MAG). Nomenclature and numbering are based on the GPATs of Arabidopsis thaliana. Reproduced, with permission, from [74].

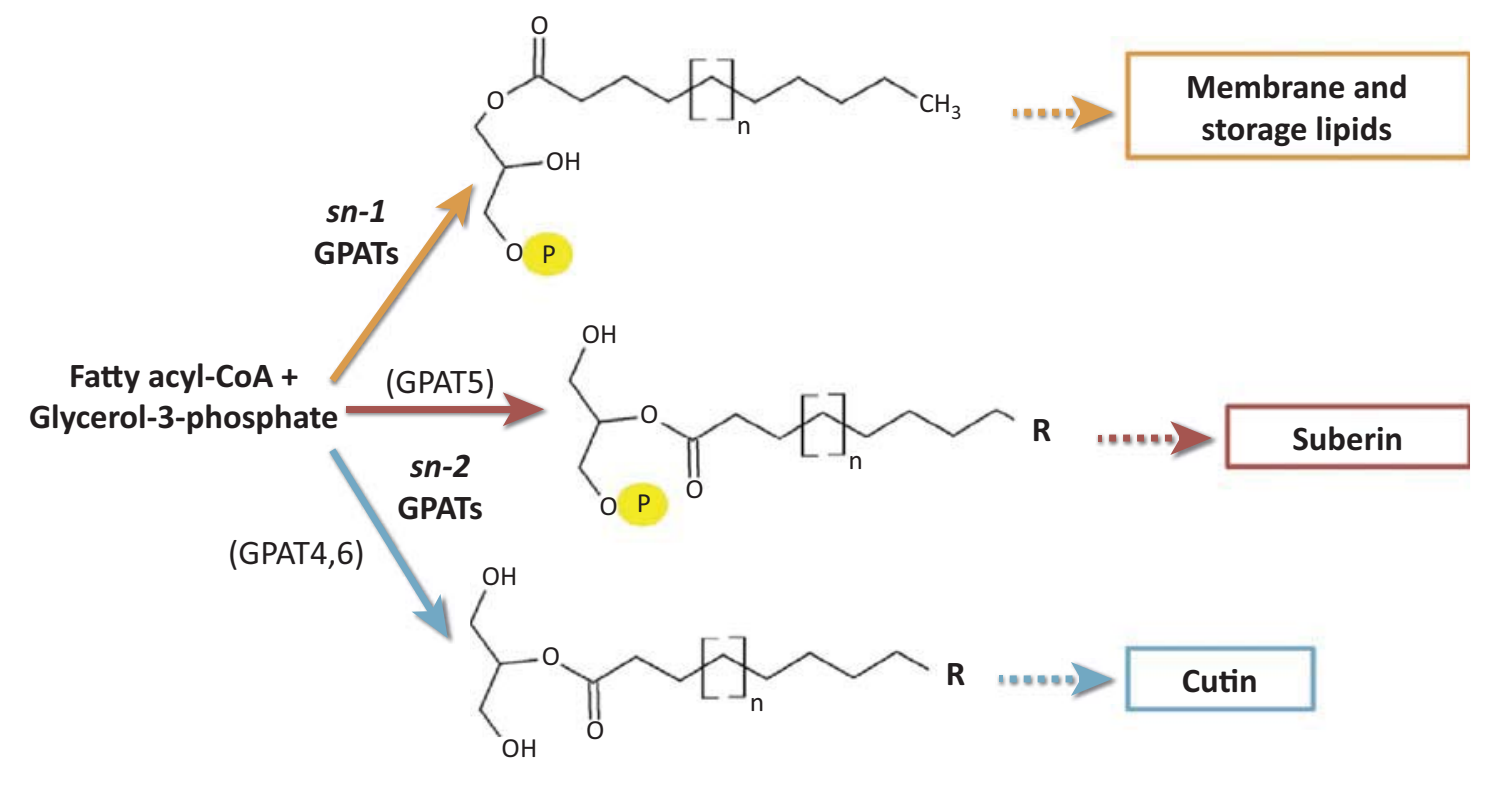

$\mathrm{R}=\mathrm{CH}_{3}, \mathrm{OH}, \mathrm{COOH}, \mathrm{OCOR}^{\prime}, \mathrm{COOR}^{\prime}$

$\overline{\text { TRENDS in Plant Science }}$

Figure I. GPATs and their role in the biosynthesis of membrane and storage lipids and of lipid polyesters.

by S-locus receptor kinase (SRK) which activates the abortion of self-pollen by a mechanism that is currently under intense debate [45-48]. The extracellular domain of SRK contains a highly conserved cysteine-rich stretch consisting of an EGF-like and a PAN_APPLE motive that are required for receptor dimerization [49], and comprises $N$ glycosylation sites that are essential for localization to the plasma membrane [50]. The genes encoding SCR protein and SRK are tightly linked in the S-locus, and occur in many allelic variants in natural plant populations [51], thus ensuring efficient cross-pollination.

Interestingly, maize roots express a closely related homolog of SRK, referred to as ZmPK1 [52]. Recent bioinformatics analysis at the genomic level revealed that the genomes of AM-competent species have a conserved SRKrelated group of receptor kinases that are absent from the non-mycorrhizal Brassicaceae [34]. The extracellular domain of these predicted AM-related receptors exhibit the canonical cysteine residues required for dimerization and several predicted $N$-glycosylation sites (Figure 2). Consistent with a role in root symbioses, the three Medicago homologs of this specific SRK-related gene family are expressed exclusively or primarily in roots (see http:// mtgea.noble.org/v3). A potential role of these AM-related receptor kinases, in addition to the established roles of leucine-rich repeat (LRR) and lysine-motive (LysM) receptor-like kinases (RLKs) [53], remains to be elucidated; however, it is intriguing to note that AM and SI may involve recognition systems that share a common evolutionary origin.

\section{Cell wall-related commonalities between symbiosis and pollen development}

Fascinating parallels have emerged from comparisons at the molecular and morphological levels between growing pollen tubes and the infection thread in root nodule symbiosis. Infection threads are invaginations of root hair cells that allow rhizobia to enter, and that guide them towards the cortex [5]. Several similarities have led to the hypothesis that the infection thread could be regarded as an inverse pollen tube that extends by a tip-growing mechanism [54]. Indeed, pollen tubes and infection threads share several closely related proteins such as pectic enzymes [55] and arabinogalactan proteins [56]. The close relationship between nodule-related and pollen-related pectic enzymes extends from the conserved coding region into the promoters. Strikingly, some promoters contain separate elements that mediate expression in flowers and nodules, respectively. Taken together, these findings strongly argue for a common evolutionary origin of cell wall-related 


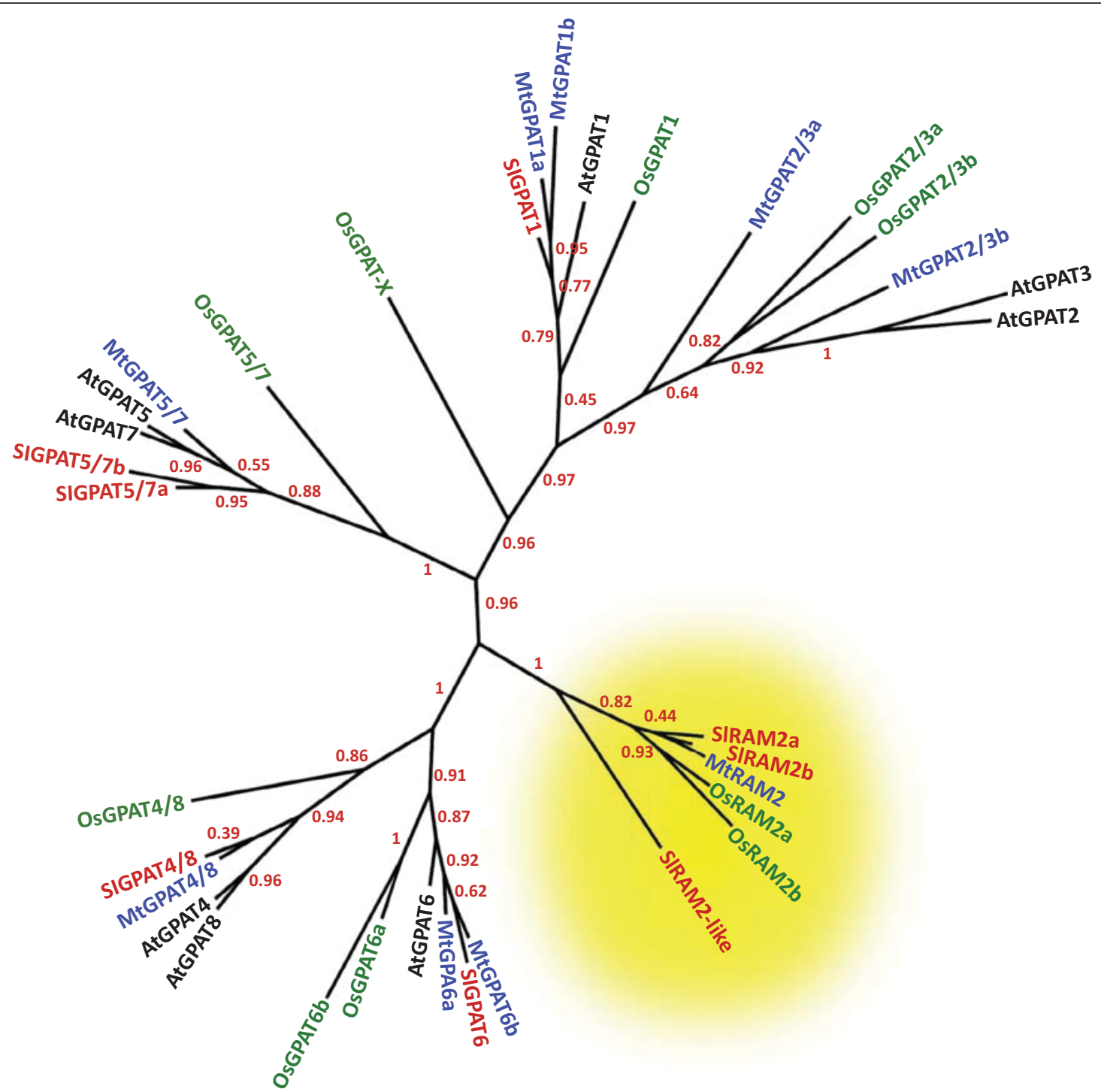

$\overline{\text { TRENDS in Plant Science }}$

Figure 1. Phylogeny of glycerol-3-phosphate acyltransferases (GPATs). Phylogram of the entire GPAT families of the AM competent species Medicago truncatula, Solanum Iycopersicum, Oryza sativa (rice), and of the non-mycorrhizal species Arabidopsis thaliana. Note that A. thaliana lacks a homolog in the RAM2 branch (yellow). Phylogenetic analysis was carried out as described using the software package at www.phylogeny.fr [73]. Support for branch separation is indicated with bootstrap values for 100 replicates.

mechanisms involved in the formation of pollen tubes and infection threads [55]. Further molecular genetic dissection of the pathways involved in these two different cellular growth phenomena will profit from such comparative studies.

\section{Evidence for overlaps from genetics}

Genetic overlaps between developmental pathways can be discovered by rescreening mutants for new phenotypes. For example, the finding that approximately half the nodulation-defective mutants in pea are also AM-defective

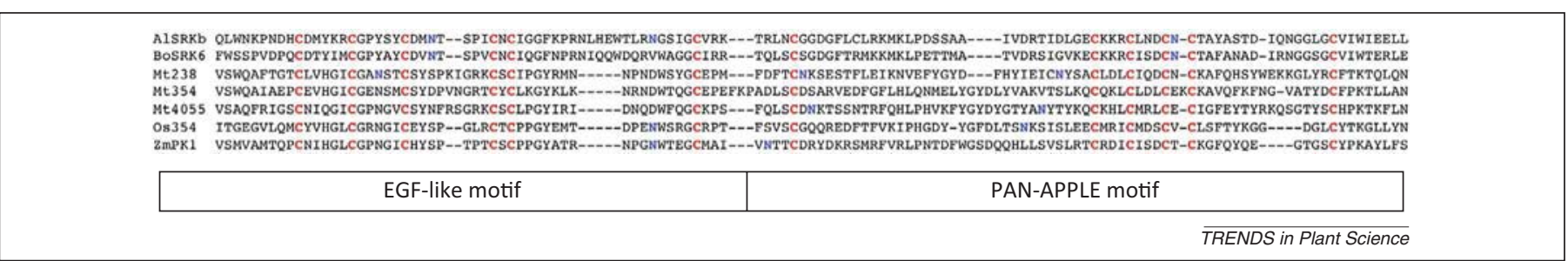

Figure 2. Conserved dimerization domain in AM-related SRK-like proteins. Conserved C-rich extracellular domain of Arabidopsis lyrata SRKb [75] and Brassica oleracea SRK6 [76], together with the three predicted Medicago truncatula AM-related SRK homologs (Mt238, Mt354, Mt4055) [34], the closest homolog in Oryza sativa (Os354), and Zea mays PK1 (ZmPK1) [52]. Conserved cysteine residues are highlighted in red, predicted $\mathrm{N}$-glycosylation sites in blue. Glycosylation sites in AISRKb were experimentally confirmed [50]. 
led to the discovery of the CSSP [57]. Applying this approach to reveal overlaps between pollen development and root symbioses appears at first sight less pertinent, and thus systematic rescreening of pollen mutants for symbiosis defects has not been carried out. Furthermore, this approach has been precluded in the cases where pollen mutants had been isolated in the non-symbiotic model species A. thaliana. Conversely, AM mutants with pollen defects should immediately be identified by their fertility defects. Indeed, some symbiosis mutants exhibit segregation distortions $[32,33,58]$ and, in the case of NUP85 and crinkle, this has been attributed to pollen defects [33,58].

Given the transcriptomic overlap between AM and flower development, ram2 and ccamk mutants could also be expected to exhibit fertility defects in addition to their symbiosis defects; however, this is not the case. This may be for two reasons: pollen is generally produced in large excess, and therefore quantitative defects in pollen fitness may be overlooked, in particular in self-fertile species. A second reason for the lack of pollen-related mutant phenotypes may be functional redundancy. For example, the secretion of components for pollen wall formation in Arabidopsis involves two ABC transporters of the G-type subfamily, ABCG1 and ABCG16, which act redundantly [59]. Similarly, RAM2 may function redundantly with other GPATs in pollen, whereas its role in mycorrhizal roots is non-redundant. Indeed, members from other branches of the GPAT tree were shown to be induced during pollen development [60] or to be required for pollen development [61-63].

\section{Concluding remarks and outlook}

The combined evidence from transcriptomics, genomics, and genetics reveals significant overlaps between root symbioses and pollen development. Components of the CSSP, including NUP85, NUP133, and CCaMK, as well as a shared mechanism involving the glycerol-3-phosphate acyltransferase RAM2, appear to function in both mycorrhizal roots and anthers. Although the shared elements may ultimately serve different downstream functions in AM and pollen development, comparison of the common elements could be informative. An important aspect of such comparative work should involve the precise transcriptomic analysis of the different cell types in anthers and mycorrhizal roots in an AM-competent species such as $M$. truncatula or rice. Gene expression data with high spatial and temporal resolution could be obtained using techniques such as cell sorting $[64,65]$ or laser-assisted micro-dissection in mycorrhizal roots $[66,67]$ and in pollen development $[68,69]$. Using such a systematic comparative approach, and exchanging information and ideas from the two research domains, could help to explain common and differential elements in these seemingly very different realms of plant biology.

\section{Acknowledgments}

We thank Patrick Favre for assistance with gene expression analysis. This work was supported by SystemsX and the Swiss National Science Foundation.

\section{References}

1 Bucher, M. et al. (2014) Through the doors of perception to function in arbuscular mycorrhizal symbioses. New Phytol. 204, 833-840
2 Gutjahr, C. and Parniske, M. (2013) Cell and developmental biology of arbuscular mycorrhiza symbiosis. Annu. Rev. Cell Dev. Biol. 29, 593-617

3 Harrison, M.J. (2012) Cellular programs for arbuscular mycorrhizal symbiosis. Curr. Opin. Plant Biol. 15, 691-698

4 Smith, S.E. and Read, D.J. (2008) Mycorrhizal Symbiosis, Academic Press

5 Oldroyd, G.E.D. et al. (2011) The rules of engagement in the legumerhizobial symbiosis. Annu. Rev. Genet. 45, 119-144

6 Singh, S. and Parniske, M. (2012) Activation of calcium- and calmodulindependent protein kinase (CCaMK), the central regulator of plant root endosymbiosis. Curr. Opin. Plant Biol. 15, 444-453

7 Gleason, C. et al. (2006) Nodulation independent of rhizobia induced by a calcium-activated kinase lacking autoinhibition. Nature 441, 1149-1152

8 Tirichine, L. et al. (2006) Deregulation of a $\mathrm{Ca}^{2+} /$ calmodulin-dependent kinase leads to spontaneous nodule development. Nature 441, 1153-1156

9 Quilichini, T.D. et al. (2014) New views of tapetum ultrastructure and pollen exine development in Arabidopsis thaliana. Ann. Bot. 114, 1189-1201

10 Ariizumi, T. and Toriyama, K. (2011) Genetic regulation of sporopollenin synthesis and pollen exine development. Annu. Rev. Plant Biol. 62, 437-460

11 Mitchell, F.J.G. (2011) Exploring vegetation in the fourth dimension. Trends Ecol. Evol. 26, 45-52

12 Alexander, T. et al. (1988) Dynamics of arbuscule development and degeneration in mycorrhizas of Triticum aestivum $\mathrm{L}$ and Avena sativa L with reference to Zea mays L. New Phytol. 110, 363-370

13 Alexander, T. et al. (1989) Dynamics of arbuscule development and degeneration in onion, bean, and tomato with reference to vesiculararbuscular mycorrhizae in grasses. Can. J. Bot. Rev. Can. Bot. 67, 2505-2513

14 Bonfante, P. and Genre, A. (2015) Arbuscular mycorrhizal dialogues: do you speak 'plantish' or 'fungish'? Trends Plant Sci. 20, 150-154

15 Murray, J.D. et al. (2013) Signaling at the root surface: the role of cutin monomers in mycorrhization. Mol. Plant 6, 1381-1383

16 Wang, E.T. et al. (2012) A common signaling process that promotes mycorrhizal and oomycete colonization of plants. Curr. Biol. 22, 2242-2246

17 Patil, S. et al. (1995) Chimeric plant calcium/calmodulin-dependent protein kinase gene with a neural visinin-like calcium-binding domain. Proc. Natl. Acad. Sci. U.S.A. 92, 4897-4901

18 Liu, Z.H. et al. (1998) Chimeric calcium/calmodulin-dependent protein kinase in tobacco: differential regulation by calmodulin isoforms. Plant Mol. Biol. 38, 889-897

19 Pandey, S. and Sopory, S.K. (1998) Biochemical evidence for a calmodulin-stimulated calcium-dependent protein kinase in maize. Eur. J. Biochem. 255, 718-726

20 Ramachandiran, S. et al. (1997) Functional domains of plant chimeric calcium/calmodulin-dependent protein kinase: regulation by autoinhibitory and visinin-like domains. J. Biochem. 121, 984-990

21 Sathyanarayanan, P.V. et al. (2000) Plant chimeric $\mathrm{Ca}^{2+} /$ calmodulindependent protein kinase - role of the neural visinin-like domain in regulating autophosphorylation and calmodulin affinity. J. Biol. Chem. $275,30417-30422$

22 Sathyanarayanan, P.V. et al. (2001) Calcium-stimulated autophosphorylation site of plant chimeric calcium/calmodulindependent protein kinase. J. Biol. Chem. 276, 32940-32947

23 Sathyanarayanan, P.V. and Poovaiah, B.W. (2002) Autophosphorylationdependent inactivation of plant chimeric calcium/calmodulin-dependent protein kinase. Eur. J. Biochem. 269, 2457-2463

24 Pandey, S. and Sopory, S.K. (2001) Zea mays CCaMK: autophosphorylation-dependent substrate phosphorylation and down-regulation by red light. J. Exp. Bot. 52, 691-700

25 Pandey, S. et al. (2002) $\mathrm{A} \mathrm{Ca}^{2+} / \mathrm{CaM}$-dependent kinase from pea is stress regulated and in vitro phosphorylates a protein that binds to AtCaM5 promoter. Eur. J. Biochem. 269, 3193-3204

26 Poovaiah, B.W. et al. (1999) Developmental regulation of the gene for chimeric calcium/calmodulin-dependent protein kinase in anthers. Planta 209, 161-171

27 Lévy, J. et al. (2004) A putative $\mathrm{Ca}^{2+}$ and calmodulin-dependent protein kinase required for bacterial and fungal symbioses. Science 303, 1361-1364 
28 Mitra, R.M. et al. (2004) $\mathrm{A} \mathrm{Ca}^{2+} /$ calmodulin-dependent protein kinase required for symbiotic nodule development: gene identification by transcript-based cloning. Proc. Natl. Acad. Sci. U.S.A. 101, 4701-4705

29 Chen, C.Y. et al. (2007) Fungal symbiosis in rice requires an ortholog of a legume common symbiosis gene encoding a $\mathrm{Ca}^{2+} /$ calmodulindependent protein kinase. Plant Physiol. 145, 1619-1628

30 Gutjahr, C. et al. (2008) Arbuscular mycorrhiza-specific signaling in rice transcends the common symbiosis signaling pathway. Plant Cell 20, 2989-3005

31 Binder, A. and Parniske, M. (2014) Analysis of the Lotus japonicus nuclear pore NUP107-160 subcomplex reveals pronounced structural plasticity and functional redundancy. Front. Plant Sci. 4, 552

32 Kanamori, N. et al. (2006) A nucleoporin is required for induction of $\mathrm{Ca}^{2+}$ spiking in legume nodule development and essential for rhizobial and fungal symbiosis. Proc. Natl. Acad. Sci. U.S.A. 103, 359-364

33 Saito, K. et al. (2007) NUCLEOPORIN85 is required for calcium spiking, fungal and bacterial symbioses, and seed production in Lotus japonicus. Plant Cell 19, 610-624

34 Favre, P. et al. (2014) A novel bioinformatics pipeline to discover genes related to arbuscular mycorrhizal symbiosis based on their evolutionary conservation pattern among higher plants. BMC Plant Biol. 14, 333

35 Chen, X. et al. (2011) sn-Glycerol-3-phosphate acyltransferases in plants. Plant Signal. Behav. 6, 1695-1699

36 Li-Beisson, Y. et al. (2013) Acyl-lipid metabolism. Arabidopsis Book 11, $\mathrm{e} 0161$

37 Yang, W.L. et al. (2010) A distinct type of glycerol-3-phosphate acyltransferase with sn-2 preference and phosphatase activity producing 2-monoacylglycerol. Proc. Natl. Acad. Sci. U.S.A. 107, 12040-12045

38 Schreiber, L. (2010) Transport barriers made of cutin, suberin and associated waxes. Trends Plant Sci. 15, 546-553

39 Geldner, N. (2013) The endodermis. Annu. Rev. Plant Biol. 64, 531-558

40 Franke, R. et al. (2000) Modified lignin in tobacco and poplar plants over-expressing the Arabidopsis gene encoding ferulate 5-hydroxylase. Plant J. 22, 223-234

41 Marita, J.M. et al. (1999) NMR characterization of lignins in Arabidopsis altered in the activity of ferulate 5-hydroxylase. Proc. Natl. Acad. Sci. U.S.A. 96, 12328-12332

42 Oldroyd, G.E.D. (2013) Speak, friend, and enter: signalling systems that promote beneficial symbiotic associations in plants. Nat. Rev. Microbiol. 11, 252-263

43 Takayama, S. and Isogai, A. (2005) Self-incompatibility in plants. Annu. Rev. Plant Biol. 56, 467-489

44 Nasrallah, J.B. and Nasrallah, M.E. (2014) S-locus receptor kinase signalling. Biochem. Soc. Trans. 42, 313-319

45 Goring, D.R. et al. (2014) The ARC1 E3 ligase promotes a strong and stable self-incompatibility response in Arabidopsis species: response to the Nasrallah and Nasrallah commentary. Plant Cell 26, 3842-3846

46 Indriolo, E. et al. (2012) The ARC1 E3 ligase gene Is frequently deleted in self-compatible Brassicaceae species and has a conserved role in Arabidopsis lyrata self-pollen rejection. Plant Cell 24, 4607-4620

47 Indriolo, E. et al. (2014) The ARC1 E3 ligase promotes two different self-pollen avoidance traits in Arabidopsis. Plant Cell 26, 1525-1543

48 Nasrallah, J.B. and Nasrallah, M.E. (2014) Robust self-incompatibility in the absence of a functional ARC1 gene in Arabidopsis thaliana. Plant Cell 26, 3838-3841

49 Naithani, S. et al. (2007) Structural modules for receptor dimerization in the S-locus receptor kinase extracellular domain. Proc. Natl. Acad. Sci. U.S.A. 104, 12211-12216

50 Yamamoto, M. et al. (2014) Site-specific N-glycosylation of the S-locus receptor kinase and its role in the self-incompatibility response of the Brassicaceae. Plant Cell 26, 4749-4762

51 Vekemans, X. et al. (2014) The evolution of selfing from outcrossing ancestors in Brassicaceae: what have we learned from variation at the S-locus? J. Evol. Biol. 27, 1372-1385

52 Walker, J.C. and Zhang, R. (1990) Relationship of a putative receptor protein kinase from maize to the S-locus glycoproteins of Brassica. Nature $345,743-746$
53 Antolin-Llovera, M. et al. (2014) Knowing your friends and foes - plant receptor-like kinases as initiators of symbiosis or defence. New Phytol. 204, 791-802

54 Szczyglowski, K. and Amyot, L. (2003) Symbiosis, inventiveness by recruitment? Plant Physiol. 131, 935-940

55 Rodriguez-Llorente, I.D. et al. (2004) From pollen tubes to infection threads: recruitment of Medicago floral pectic genes for symbiosis. Plant J. 39, 587-598

56 Nguema-Ona, E. et al. (2012) Arabinogalactan proteins in root and pollen-tube cells: distribution and functional aspects. Ann. Bot. 110, 383-404

57 Duc, G. et al. (1989) First report of non-mycorrhizal plant mutants (myc-) obtained in pea (Pisum sativum L.) and fababean (Vicia faba L.). Plant Sci. 60, 215-222

58 Tansengco, M.L. et al. (2004) Pollen development and tube growth are affected in the symbiotic mutant of Lotus japonicus, crinkle. Plant Cell Physiol. 45, 511-520

59 Yadav, V. et al. (2014) ABCG transporters are required for suberin and pollen wall extracellular barriers in Arabidopsis. Plant Cell 26, $3569-3588$

60 Cnudde, F. et al. (2006) Changes in gene expression during male meiosis in Petunia hybrida. Chromosome Res. 14, 919-932

61 Chen, X. et al. (2014) Glycerol-3-phosphate acyltransferase 4 is essential for the normal development of reproductive organs and the embryo in Brassica napus. J. Exp. Bot. 65, 4201-4215

$62 \mathrm{Li}, \mathrm{X}$-C. et al. (2012) Glycerol-3-phosphate acyltransferase 6 (GPAT6) is important for tapetum development in Arabidopsis and plays multiple roles in plant fertility. Mol. Plant 5, 131-142

63 Zheng, Z.F. et al. (2003) Arabidopsis AtGPAT1, a member of the membrane-bound glycerol-3-phosphate acyltransferase gene family, is essential for tapetum differentiation and male fertility. Plant Cell $15,1872-1887$

64 Birnbaum, K. et al. (2003) A gene expression map of the Arabidopsis root. Science 302, 1956-1960

65 Birnbaum, K. et al. (2005) Cell type-specific expression profiting in plants via cell sorting of protoplasts from fluorescent reporter lines. Nat. Methods 2, 615-619

66 Berruti, A. et al. (2013) Application of laser microdissection to identify the mycorrhizal fungi that establish arbuscules inside root cells. Front. Plant Sci. 4, 135

67 Gomez, S.K. and Harrison, M.J. (2009) Laser microdissection and its application to analyze gene expression in arbuscular mycorrhizal symbiosis. Pest Manag. Sci. 65, 504-511

68 Aya, K. et al. (2011) Comprehensive network analysis of antherexpressed genes in rice by the combination of 33 laser microdissection and 143 spatiotemporal microarrays. PLoS ONE 6, e26162

69 Suwabe, K. et al. (2008) Separated transcriptomes of male gametophyte and tapetum in rice: validity of a laser microdissection (LM) microarray. Plant Cell Physiol. 49, 1407-1416

70 Harper, J.F. and Harmon, A. (2005) Plants, symbiosis and parasites: A calcium signalling connection. Nat. Rev. Mol. Cell Biol. 6, 555-566

71 Poovaiah, B.W. et al. (2013) Recent advances in calcium/calmodulinmediated signaling with an emphasis on plant-microbe interactions. Plant Physiol. 163, 531-542

72 Delaux, P-M. et al. (2014) Comparative phylogenomics uncovers the impact of symbiotic associations on host genome evolution. PLoS Genet. 10, e1004487

73 Dereeper, A. et al. (2008) Phylogeny.fr: robust phylogenetic analysis for the non-specialist. Nucleic Acids Res. 36, W465-W469

74 Beisson, F. et al. (2012) Solving the puzzles of cutin and suberin polymer biosynthesis. Curr. Opin. Plant Biol. 15, 329-337

75 Kusaba, M. et al. (2001) Self-incompatibility in the genus Arabidopsis: characterization of the S-locus in the outcrossing A. lyrata and its autogamous relative A. thaliana. Plant Cell 13, 627-643

76 Stein, J.C. et al. (1991) Molecular cloning of a putative receptor protein kinase gene encoded at the self-incompatibility locus of Brassica oleracea. Proc. Natl. Acad. Sci. U.S.A. 88, 8816-8820 